\title{
Dynamic facial expressions of emotion induce representational momentum
}

\author{
Sakiko Yoshikawa and Wataru SATo \\ Kyoto University, Kyoto, Japan
}

\begin{abstract}
Two experiments were conducted to explore whether representational momentum (RM) emerges in the perception of dynamic facial expression and whether the velocity of change affects the size of the effect. Participants observed short morphing animations of facial expressions from neutral to one of the six basic emotions. Immediately afterward, they were asked to select the last images perceived. The results of the experiments revealed that the RM effect emerged for dynamic facial expressions of emotion: The last images of dynamic stimuli that an observer perceived were of a facial configuration showing stronger emotional intensity than the image actually presented. The more the velocity increased, the more the perceptual image of facial expression intensified. This perceptual enhancement suggests that dynamic information facilitates shape processing in facial expression, which leads to the efficient detection of other people's emotional changes from their faces.
\end{abstract}

Among the social signals conveyed by the face, dynamic changes in emotional facial expressions indicate current mood and moment-to-moment changes in the emotional state of individuals. Over the past few decades, a large number of empirical studies have examined the psychological mechanisms that are involved in processing facial expression, and a wealth of findings has been accumulated (Ekman, 1993). More recently, these studies have been complemented by brain science research, and it is becoming clear that a variety of brain areas are involved in processing facial expression (Haxby, Hoffman, \& Gobbini, 2000). However, most studies on the recognition of facial expressions - in both psychology and neuroscience - have used static images of facial expressions. Consequently, little is known about the perceptual processes that are involved in processing dynamic facial expressions of emotion.

Recent neuroscience and lesion research provides some clues in this regard. An fMRI study (Sato, Kochiyama, Yoshikawa, Naito, \& Matsumura, 2004) reported that several cortical visual areas, such as inferior occipital gyri, middle temporal gyri, and fusiform gyri showed greater activation for dynamic facial expressions than for static ones. These brain areas comprise the main neural substrates for visual processing of faces (Haxby et al., 2000). A lesion study (de Gelder, Vroomen, Pourtois, \& Weiskrantz, 1999), which reported evidence that a blindsight patient could recognize facial expressions presented in his blind field, showed that the correct score of response was higher for dynamic video stimuli than for static stimuli (Experiment 3). This result indicates that even facial expression processing via the subcortical route, such as the superior colliculus and pulvinar, reflects a difference be- tween the dynamic versus static properties of the stimuli. This implies that the influence of the dynamic properties of facial expression would emerge at very early stages of perceptual processing.

Considering the data from neuroscientific literature that have been obtained so far, we assumed that dynamic information would have an influence on the perceptual processing of facial expressions. In this article, we explore whether representational momentum (RM; Freyd, 1987; Freyd \& Finke, 1984) emerges in the perception of dynamic facial expression. RM has been reported in the context of motion perception literature, describing a phenomenon in which the final position of an object - when seen to be actually moving or changing position in a way that implies continuous motion-shifts in the perceiver's mind in the direction of the observed transformation (Freyd \& Finke, 1984; Hubbard, 1990). Since its discovery, this effect has been found in several types of implied change of object shape (Kelly \& Freyd, 1987). However, whether the RM effect emerges when perceiving dynamic facial expressions of emotion remains unknown. In the present study, we aimed to demonstrate RM in dynamic facial expressions of emotion. Specifically, we predicted that when a person perceives a dynamic facial expression changing from a neutral state to a particular emotional expression, such as anger or happiness, the last image of the dynamic sequence will be perceived in an exaggerated form, showing stronger emotional intensity.

We also manipulated the speed with which the facial expressions changed in order to examine the effect of velocity. Previous research on RM for implied target movement has revealed a robust effect of velocity - namely, that faster velocity of target movement leads to larger for- 
ward displacement (see, e.g., Hubbard, 2005; Hubbard \& Bharucha, 1988). On the basis of this observation, we generated the following working hypothesis: As the velocity of change in facial expression increases, observers perceive the facial expression to be showing stronger emotional intensity.

Two methodological points in the present research need to be mentioned. First, we used pseudonatural dynamic facial expressions that were created by a morphing technique rather than natural facial expressions. We used morphing animation because it allowed us to create well-controlled experimental materials. Natural facial expression stimuli captured on video clips are not appropriate for investigating basic perceptual processes because they include several uncontrollable factors, such as head movement, idiosyncratic muscle movement, and eye blinking. Morphing animation of facial expression has several merits for recognition research. It is free from the uncontrollable factors mentioned above, easy to control in terms of the combination and duration of the stimuli, and looks so natural that observers believe that they are looking at real faces.

The second characteristic of our research that requires mention is the nature of the task, which is best described as an interactive image-selection task (Benson \& Perrett, 1991). In this task, participants observed a short animation stimulus of a facial expression moving from neutral to one of the six basic emotions. Immediately afterward, participants were asked to select the last image perceived by using a slider on the computer display. This procedure made it possible to produce the images participants thought they had perceived, with little constraint. When the images had been selected, participants viewed the animation stimuli again and had the opportunity to modify their original choice of image. Unlike typical RM paradigms in which participants match their internal image with a limited number of predetermined images, this interactive image-selection procedure enabled us to obtain participants' internal images more precisely. A potential drawback of this procedure might have been the decay of perceived images over time. In order to avoid this procedural shortcoming, we presented the same dynamic stimulus twice. In the second exposure, participants perceptu- ally matched the image that they had selected with the last image of the stimulus and modified their choice if they thought the two images did not match exactly.

\section{EXPERIMENT 1}

\section{Method}

Participants. Sixteen university students (10 male and 6 female) participated in the experiment. The average age was 21.3 years old. All had normal or corrected-to-normal vision.

Experimental design. The experiment was constructed as a within-subjects two-factorial design, with velocity $(20 \mathrm{msec} /$ frame, $40 \mathrm{msec} / \mathrm{frame}$, and static) and emotion (anger, disgust, fear, happiness, sadness, and surprise) as the factors.

Stimuli. From Ekman and Friesen's (1976) set of facial images, we selected one neutral expression slide and six emotional expression slides for each of four individuals (two men and two women). All of the face slides were digitized into $300 \times 200$ pixel images. Using computer-morphing techniques (Mukaida et al., 2000), 24 intermediate images between the neutral expression $(0 \%)$ and each of the six emotional expressions (100\%) were produced in $4 \%$ steps. In order to produce dynamic facial expression stimuli in which the faces changed from neutral to one of the six emotional expressions, we presented a total of 26 frames of images in quick succession; that is, 1 neutral image, 24 intermediate images, and an original $100 \%$ emotional image. Figure 1 shows the first, some intermediate, and last images of a dynamic stimulus. The stimulus duration was $520 \mathrm{msec}$ (20 msec/frame) and 1,040 $\mathrm{msec}(40 \mathrm{msec} /$ frame). A static condition - in which the images of $100 \%$ emotional expression were presented for $1,040 \mathrm{msec}$ - was included as a control. The images of $100 \%$ emotional expression corresponded to the original image from Ekman and Friesen. The stimuli durations for Experiment 1 were decided by referring to Hara and Kobayashi (1995), who had analyzed the time course of video-recorded faces expressing the six basic emotions and reported their onset to peak duration. With the shortest durations for surprise and anger and the longest for sadness, their ranges were approximately $330-1,400 \mathrm{msec}$.

One point to note is the naturalness of these linearly changing morphing expressions. In a previous study (Sato \& Yoshikawa, 2004), in which participants rated the naturalness of morphing animation stimuli on a 7-point scale, the mean naturalness rating for all stimuli-irrespective of velocity - exceeded 4 (moderately natural), and none of the participants noticed that the stimuli were artificial animations created from static images. Given this evidence, we proceeded on the basis that morphing animations provide adequate stimuli for examining perceptual processing of dynamic facial expressions of emotion.

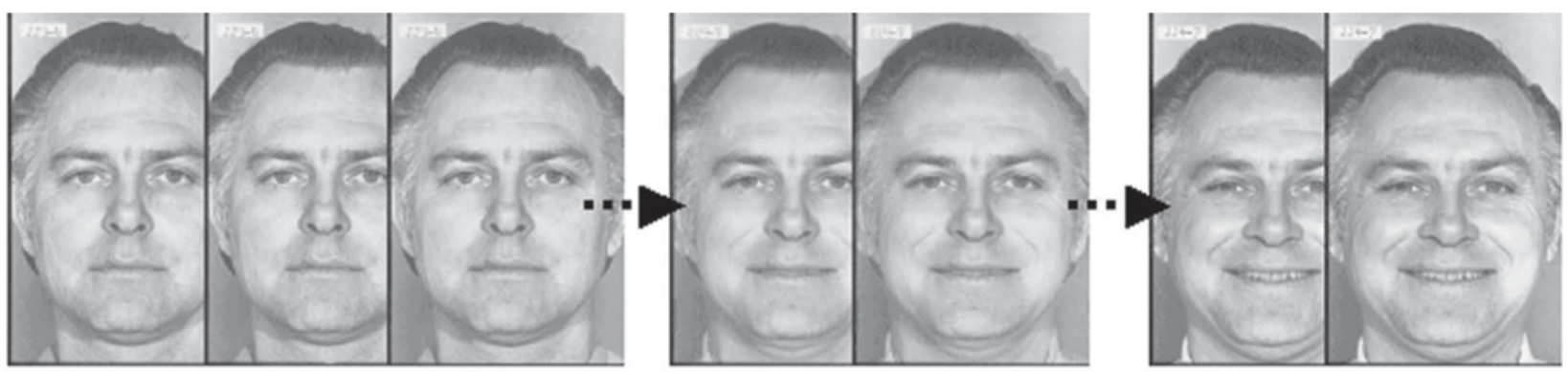


Apparatus. Stimulus presentation and response recording were controlled by a program written in Visual $\mathrm{C}++5.0$ (Microsoft, 1997) and implemented on a Windows computer. The stimuli were presented on a 17-in. flat monitor (Eizo T550) from a viewing distance of $0.57 \mathrm{~m}$.

Procedure. Figure 2 shows the sequence of an experimental trial. The left window on the monitor was for the stimulus presentation, and the right window was for the participants' responses. The visual angles of the stimulus and response windows were $11.1^{\circ} \times 7.8^{\circ}$, respectively. For each trial, $250 \mathrm{msec}$ after the presentation of the dynamic stimulus, the initial face image appeared in the response window. Participants were instructed to match exactly the image on the response window with the last image of the dynamic stimulus by dragging a slider to either the left or right with the mouse. The initial image in the response window was a face with an emotional expression of $80 \%, 90 \%$, or $100 \%$ intensity. Three ranges of the slider scale were predefined, each of which covered a range of $100 \%$ (i.e., $30 \%-130 \%, 40 \%-140 \%$, and $50 \%-150 \%$ of emotional intensity). The range of the slider scale varied randomly across trials, and slider ranges were not visible to participants. After selecting the image, participants clicked any key on

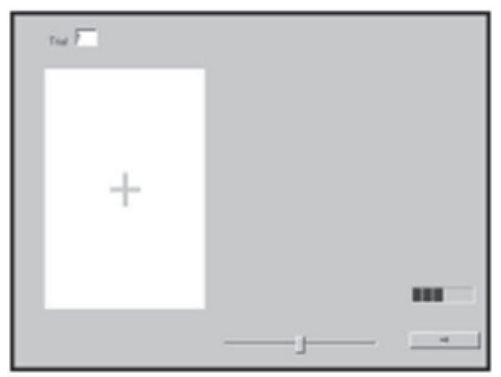

\section{Warning} signal
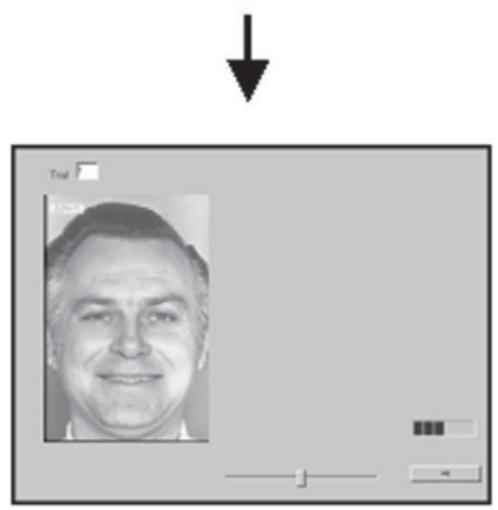

Presentation of the stimuli

\section{$\downarrow|S|=250 \mathrm{msec}$}

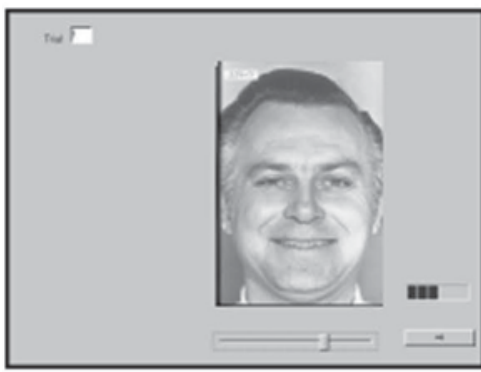

Subject's

first try

(producing the

same image

as that in the

last frame)

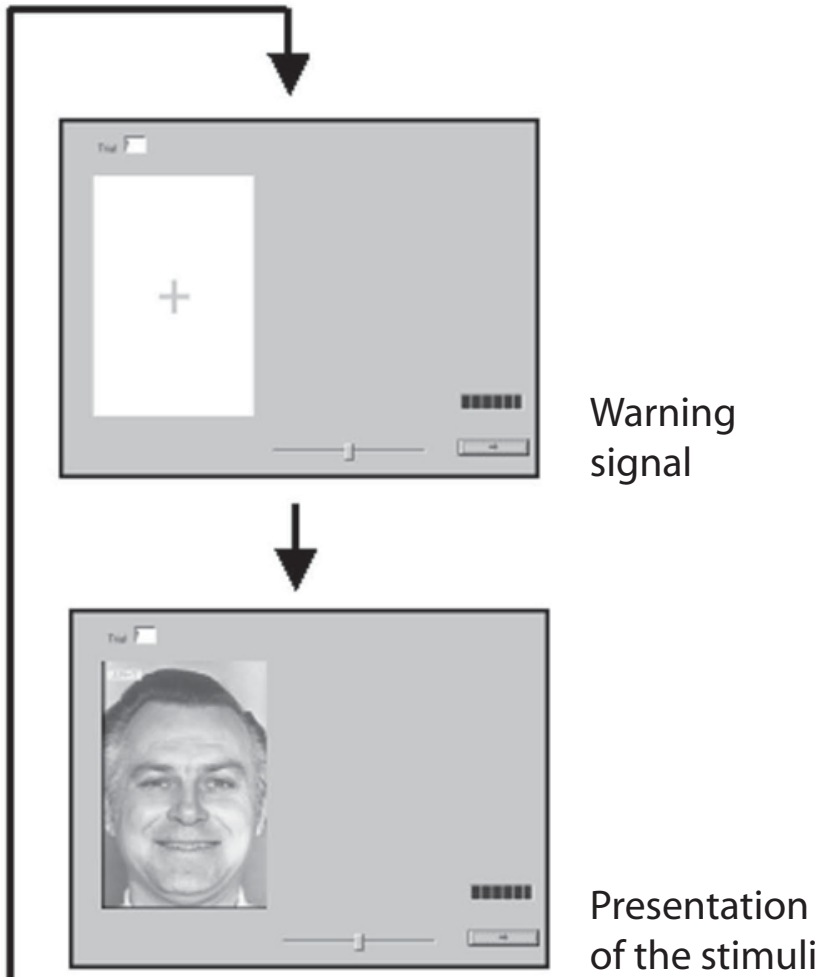

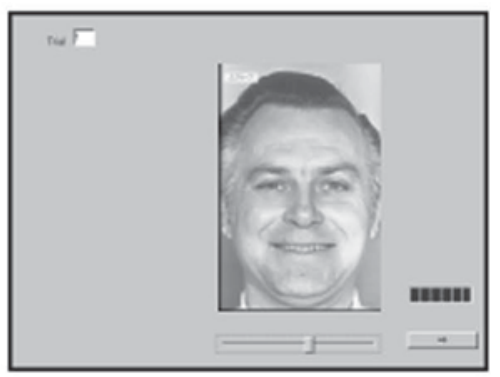

Subject's second try (check and adjust the image if necessary)

Figure 2. The sequence of an experimental trial. 
the keyboard, and the image in the response window disappeared. The stimulus was then presented again in the left window and, $250 \mathrm{msec}$ after its presentation, the face image chosen by the participant appeared in the response window. If the participants thought that they matched, they clicked the "next" button on the display and went on to the next trial. If they thought that they did not match, then the participants modified the image in the response window using the slider. ${ }^{1} \mathrm{~A}$ total of 48 trials ( 24 trials of $20-\mathrm{msec} /$ frame and 24 trials of $40-\mathrm{msec} /$ frame conditions) were performed in blocks, and the order of administration was counterbalanced across participants. Before starting the experimental trials, participants were given several practice trials, and they practiced image manipulation by a slider using a mouse. After completing the experimental conditions, 24 control trials were conducted, in which the static image of $100 \%$ emotional expression was presented for 1,040 msec. Participants selected the image in the same way as they had in the dynamic stimuli condition. Completion of all the tasks took about $15 \mathrm{~min}$.

Data analysis. The data were analyzed using SPSS 10.0J (SPSS Japan). The percentage of the images was analyzed with a 3 (velocity) $\times 6$ (emotion) repeated measures ANOVA. On the measures that violated spherical assumptions $(p<.1)$, the degree of freedom was adjusted using the Greenhouse-Geisser method. As follow-up analyses, pairwise comparisons between each dynamic condition and the static condition were performed using the Dunnett method. The results of all tests were considered statistically significant at $p<.05$.

\section{Results}

The results of the experiment are shown in Figure 3 (left panel). The figure shows the mean percentage of images in each condition and their standard errors of the means. The average percentages were $102.6 \%, 99.4 \%$, and $98.9 \%$ for the $20-\mathrm{msec} /$ frame, $40-\mathrm{msec} /$ frame, and static conditions, respectively. If participants had precisely reproduced the last image, then the bar of the figure would have indicated $100 \%$.

The ANOVA on the percentage of the images showed a significant main effect of velocity $[F(2,30)=4.11, p<$ $\left..05, \eta^{2}=.215\right]$. The main effect of emotion and the interaction of velocity $\times$ emotion were not statistically significant $[F \mathrm{~s}<2.2, p \mathrm{~s}>.1]$.

Follow-up multiple comparisons on the main effect of velocity revealed a significant difference between the 20$\mathrm{msec} /$ frame and static conditions $(p<.05)$.

\section{Discussion}

The results showed that in the $20 \mathrm{msec} /$ frame condition, the RM effect emerged. This means that when seeing a dynamic facial expression changing at a fast-enough velocity, we perceive a more intensified emotional image than what actually appears.

Nevertheless, we should also point out that the size of the RM effect was not very large, even though it was statistically significant. There are a couple of possible reasons for this. First, the velocity of the dynamic stimuli that were used was not appropriate for RM to emerge. Although a previous encoding study reported that participants naturally made facial expressions of emotion at about the same velocity as that used in the present study (Hara \& Kobayashi, 1995), this does not necessarily mean that the velocity was adequate for an examination of representational momentum. Second, there is a possibility that the
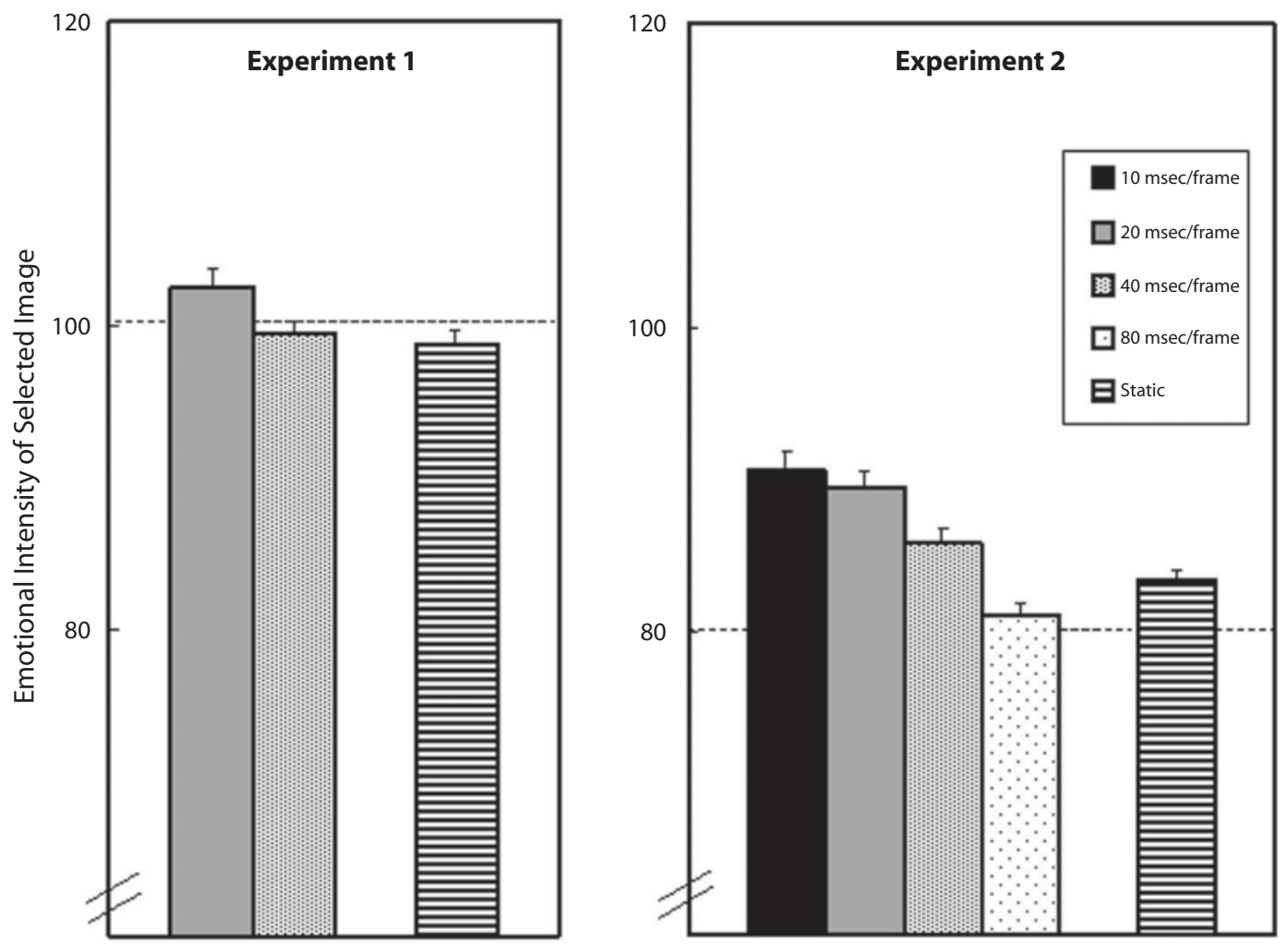

Figure 3. The mean percentage of the images in each condition of Experiment 1 and Experiment 2, shown with the standard error of the mean. 
last frames of dynamic stimuli used in the experiment exhibited such high emotional intensity that a kind of ceiling effect occurred. The original (i.e., 100\%) images that were used in the present experiment were the facial expressions arrived at when the models moved their facial muscles to their maximum level (Ekman \& Friesen, 1976). In normal circumstances, people may seldom come across these extreme emotional expressions (Russell, 1997), and when they do, they would see them as the peak of a changing sequence and, therefore, at close to zero velocity.

We speculated that these factors might impose some perceptual constraints and yield an inhibitory effect for RM. In order to examine these possibilities, we conducted Experiment 2 , adding two more velocity conditions and using less intense emotional expressions ( $80 \%$ intensity of the original image) as the objective last image of the dynamic stimuli.

Another possibility that might have influenced the size of RM was the anchoring effect induced by the initial face image that appeared in the response window. In Experiment 1, the face image was the same as the last images of the dynamic and static stimuli for one third of the time. For two thirds of the time, it was an image that was $90 \%$ or $80 \%$ of the last images of the dynamic and static facial stimuli. If response performance is anchored by the face image seen in the response window, then the small RM in Experiment 1 could be due to the fact that the response window displayed faces that were always at or behind the memory point. In Experiment 2, we modified the procedure in order to cancel out this possibility.

\section{EXPERIMENT 2}

\section{Method}

Participants. Twelve university students (all male) took part in the experiment. The average age was 22.3 years old. None had participated in Experiment 1. All had normal or corrected-to-normal vision.

Experimental design. The experiment was constructed as a withinsubjects two-factorial design, with velocity $(10 \mathrm{msec} / \mathrm{frame}, 20 \mathrm{msec} /$ frame, $40 \mathrm{msec} /$ frame, $80 \mathrm{msec} /$ frame, and static) and emotion (anger, disgust, fear, happiness, sadness, and surprise) as the factors.

Stimuli. The stimuli used in Experiment 2 were essentially the same as those in Experiment 1, except that 21 frames (from $0 \%$ to $80 \%$ ) were used instead of 26 frames. Four different velocity conditions were administered: $210 \mathrm{msec}$ (10 msec/frame), $420 \mathrm{msec}$ (20 msec/frame), $840 \mathrm{msec}$ (40 $\mathrm{msec} /$ frame), and 1,680 $\mathrm{msec}$ ( $80 \mathrm{msec} / \mathrm{frame})$. Additionally, a static condition was prepared in which the $80 \%$ intensity images of each emotional expression were presented for $840 \mathrm{msec}$.

Apparatus. The apparatus used for Experiment 2 was the same as that used in Experiment 1.

Procedure. The initial image in the response window was a face with an emotional expression at $70 \%, 80 \%$, or $90 \%$ intensity. Three ranges of slider scale were predefined, each of which covered a range of $100 \%$ (i.e., $20 \%-120 \%, 30 \%-130 \%$, and $40 \%-140 \%$ emotional intensity). The range of the slider scale varied randomly across trials. Apart from the change of stimuli and the additional velocity conditions, the main procedure was essentially the same as that in Experiment 1 . Participants performed 24 trials for each velocity condition, and the order of the four velocity conditions was counterbalanced across participants. The static condition was administered after the four dynamic facial expression conditions. Participants took about 30 min to complete the tasks.

Data analysis. An ANOVA and follow-up multiple comparisons were conducted using methodology identical to that used in Experi- ment 1 . In addition, for the follow-up analyses of velocity factor, post hoc trend analysis using the Scheffé method was conducted in order to investigate the profiles of the changes across velocity levels (for the four velocity conditions, but excluding the static condition).

\section{Results}

Figure 3 (right panel) shows the mean percentage of the images participants selected for each emotion and velocity condition and their standard errors of the means. The average percentages were $90.6 \%, 89.5 \%, 85.8 \%, 81.1 \%$, and $83.4 \%$ for the $10-\mathrm{msec} /$ frame, $20-\mathrm{msec} /$ frame, $40-\mathrm{msec} /$ frame, $80-\mathrm{msec} /$ frame, and static conditions, respectively. If participants precisely reproduced the last image, then the percent of the image would be $80 \%$.

The ANOVA revealed significant main effects of velocity $\left[F(4,44)=7.38, p<.001, \eta^{2}=.40\right]$ and emotion $\left[F(5,55)=6.94, p<.001, \eta^{2}=.39\right]$. There was no significant interaction between these two factors $(F<1.3$, $p>.1)$.

For the main effect of velocity, pairwise comparisons with each dynamic condition and the static condition indicated that the percentages of image in the 10-msec/frame and 20 $\mathrm{msec} /$ frame conditions were higher than those in the static condition $(p \mathrm{~s}<.05)$. There were no significant differences between the static condition and both 40- and 80-msec/frame conditions. For this main effect, a post hoc trend analysis revealed a linear trend for increase of percentage as a function of velocity $[F(1,11)=22.17, p<.001]$.

\section{Discussion}

The results of Experiment 2 clearly indicated that when perceiving dynamic facial expressions of emotion, participants perceived intensified versions of the last images. The degree of deviation of the perceived image from the presented image was proportional to velocity. The faster the movement, the more intensified the emotional images were perceived as being.

Another conspicuous result was the difference of the size of the RM effect between Experiments 1 and 2. Because the $20-\mathrm{msec} /$ frame and $40-\mathrm{msec} /$ frame conditions were included in both Experiments 1 and 2, a direct comparison of the size of the RM effect is possible for these conditions. The effect was more evident in Experiment 2 than in Experiment 1, suggesting that the emotional intensity of the last image was a crucial factor in determining the size of the RM effect. The interpretation of this point will be discussed in the General Discussion.

In Experiment 2, the main effect was significant not only for velocity, but also for emotion. However, it is not clear at present whether the resultant differences between emotional categories can be attributed to the effect of emotion per se, or whether they reflect differences in the visual properties of each emotional stimulus. Further discussion of this point is beyond the scope of this article.

\section{GENERAL DISCUSSION}

The present research clearly demonstrated the emergence of RM in the perception of dynamic facial expressions. To our knowledge, this is the first demonstration 
of the RM effect in the perception of dynamic facial expressions. The last images of the dynamic stimuli in all velocity conditions were the same: $100 \%$ (Experiment 1 ) or $80 \%$ (Experiment 2) emotional intensity frames. Nevertheless, the perceived images were different across velocity conditions, and the RM effect was stronger when the facial expression changed more rapidly. This evidence of the RM effect suggests that at the stage of visual processing, motion information closely interacts with shape information, which leads to the perception of transformed (i.e., intensified emotional) images when processing dynamic facial expressions.

The emotional intensity of the last images of the dynamic stimuli influenced the size of the RM effect. The last images were 100\% intensity in Experiment 1 and 80\% in Experiment 2. The resultant RM effect was much larger in Experiment 2 than in Experiment 1. This implies that the RM effect obtained was not yielded on the basis of lower level sensory processes. An informal survey after the experiments revealed that participants had not noticed that the images were artificial and made by computer manipulation - not even those images that exceeded $100 \%$. Hence, it seems difficult to explain this difference as a response bias that was due to participants' intentional avoidance of selecting unnaturally intensified images.

As was mentioned earlier, the last images of the dynamic stimuli in Experiment 1 were those in which the models moved their facial muscles at maximum level, and it is quite rare for us to see such extreme emotional expressions in everyday social interaction (Russell, 1997). When we do come across them, they may appear as a peak expression of a changing sequence with close to zero velocity. It may be that this experiential factor influences perception, leading to a suppression of the emergence of the RM effect.

A recent fMRI study (Sato et al., 2004) gives some clues about the neural mechanisms that may be involved in the RM effect of dynamic facial expression. In this study, dynamic emotional expressions were presented, and static images of emotional expression and dynamic mosaics were used as controls. The analysis of brain activation indicated that, in comparison with both static facial expressions and dynamic mosaics, higher activations were observed for dynamic emotional expressions in the broad brain regions including the inferior occipital gyri, the superior temporal sulci (STS), the amygdala, the inferior parietal lobule, and the premotor cortex.

Activation of the STS for dynamic facial expressions is of particular interest, considering the evidence from other neuroscience literature. This region is known to be active in response to various types of biological motion stimuli, such as movements of the eyes, mouth, hands, and body (Allison, Puce, \& McCarthy, 2000; Puce, Allison, Bentin, Gore, \& McCarthy, 1998). The STS is also related to the perception of implied motion in static images (Kourtzi \& Kanwisher, 2000; Senior et al., 2000), which is known to be another type of RM phenomenon (Freyd, 1987). A typical example is that when perceiving a static image of an object undergoing unidirectional movement — such as a person jumping off a wall - people remember the position of a person as being further along the path of movement. The RM effect that emerges when perceiving dynamic stimuli and when perceiving implied motion have in common a distortion of visuospatial information caused by motion. Researchers have reported that in both monkey and human visual cortices, the STS receives input from both the ventral object recognition system and the dorsal spatial location-movement system (Oram \& Perrett, 1996). This functional anatomy suggests that the STS integrates information about motion and shape. Taken together, the available evidence from the recent neurophysiological literature seems to support the view that neural pathways involving the STS are crucial for the RM effect in perceiving dynamic facial expression.

In summary, the present results indicate that the RM effect that occurred when perceiving dynamic facial expression and velocity of the movement affected the size of the effect. These results suggest that we possess a cognitive mechanism that makes it possible to efficiently recognize sudden changes of emotional state in others in a very short period of time. Perceiving stronger emotion from rapid changes of facial expression has high ecological value and is beneficial for social interaction. Having this kind of perceptual mechanism makes it easier to predict the subsequent behavior a person will take. Rapid changes of facial expression in others may also signal that something new or unexpected has happened in the environment, which also provides useful clues for an observer when interacting with other people. Psychological mechanisms for detecting these signals efficiently would be valuable for higher organisms - especially for those living in societies, as humans do.

\section{AUTHOR NOTE}

This study was supported by special coordination funds for promoting understanding of science and technology by the Japan Science and Technology Agency. Correspondence should be directed to S. Yoshikawa, Kokoro Research Center, Kyoto University, Yoshida-honmachi, Sakyoku, Kyoto 606-8501, Japan (e-mail: say@educ.kyoto-u.ac.jp).

Note-This article was accepted by the previous editorial team, when John Jonides was Editor.

\section{REFERENCES}

Allison, T., Puce, A., \& McCarthy, G. (2000). Social perception from visual cues: Role of the STS region. Trends in Cognitive Sciences, 4 , 267-278.

Benson, P. J., \& Perrett, D. I. (1991). Perception and recognition of photographic quality facial caricatures: Implications for the recognition of natural images. European Journal of Cognitive Psychology, 3, 105-135.

de Gelder, B., Vroomen, J., Pourtois, G., \& Weiskrantz, L. (1999). Non-conscious recognition of affect in the absence of striate cortex. NeuroReport, 10, 3759-3763.

Ekman, P. (1993). Facial expression and emotion. American Psychologist, 48, 384-392.

Ekman, P., \& Friesen, W. V. (1976). Pictures of facial affect. Palo Alto, CA: Consulting Psychologists Press.

FreYd, J. J. (1987). Dynamic mental representations. Psychological Review, 94, 427-438.

FreYd, J. J., \& Finke, R. A. (1984). Representational momentum. Journal of Experimental Psychology: Learning, Memory, \& Cognition, 10, 126-132.

Hara, F., \& Kobayashi, H. (1995). Experimental research on dynamic 
recognition system for human facial expressions: Development of recognition and generation system for facial expression. In Psychological and informatics research on Kansei information processing (Research Report of MEXT Grants-in-Aid for Scientific Research in Priority Areas, pp. 275-280). Tokyo: MEXT.

Haxby, J. V., Hoffman, E. A., \& Gobbini, M. I. (2000). The distributed human neural system for face perception. Trends in Cognitive Sciences, 4, 223-233.

Hubbard, T. L. (1990). Cognitive representation of linear motion: Possible direction and gravity effects in judged displacement. Memory \& Cognition, 18, 299-309.

Hubbard, T. L. (2005). Representational momentum and related displacements in spatial memory: A review of the findings. Psychonomic Bulletin \& Review, 12, 822-851.

Hubbard, T. L., \& Bharucha, J. J. (1988). Judged displacement in apparent vertical and horizontal motion. Perception \& Psychophysics, 44, 211-221.

Kelly, M. H., \& Freyd, J. J. (1987). Explorations of representational momentum. Cognitive Psychology, 19, 369-401.

Kourtzi, Z., \& Kanwisher, N. (2000). Activation in human MT/MST by static images with implied motion. Journal of Cognitive Neuroscience, 12, 48-55.

Mukaida, S., Kamachi, M., Kato, T., Oda, M., YoshiKawa, S., \& Akamatsu, S. (2000). Foolproof utilities for facial image manipulation [Unpublished computer software]. Kyoto: ATR.

Oram, M. W., \& Perrett, D. I. (1996). Integration of form and motion in the anterior superior temporal polysensory area (STPa) of the macaque monkey. Journal of Neurophysiology, 76, 109-126.

Puce, A., Allison, T., Bentin, S., Gore, J. C., \& McCarthy, G.
(1998). Temporal cortex activation in humans viewing eye and mouth movements. Journal of Neuroscience, 18, 2188-2199.

Russell, J. A. (1997). Reading emotions from and into faces: Resurrecting a dimensional-contextual perspective. In J. A. Russell \& J.-M. Fernández-Dols (Eds.), The psychology of facial expression (pp. 295-320). Cambridge: Cambridge University Press.

Sato, W., Kochiyama, T., Yoshikawa, S., Naito, E., \& MatsuMURA, M. (2004). Enhanced neural activity in response to dynamic facial expressions of emotion: An fMRI study. Cognitive Brain Research, 20, 81-91.

Sato, W., \& YoshikaWa, S. (2004). The dynamic aspects of emotional facial expressions. Cognition \& Emotion, 18, 701-710.

Senior, C., Barnes, J., Giampietro, V., Simmons, A., Bullmore, E. T., Brammer, M., \& David, A. S. (2000). The functional neuroanatomy of implicit-motion perception or representational momentum. Current Biology, 10, 16-22.

\section{NOTE}

1. In both Experiments 1 and 2, we adopted the procedure in which the participants were given a second chance to modify the image they had chosen at the first presentation if they felt that it did not match well upon observing the stimuli at the second presentation. Under this procedure, the participants modified the image on about half of the trials $(51.5 \%)$, and the mean size of the modification was $0.7 \%(S D \pm 6.8)$.

(Manuscript received May 5, 2006; revision accepted for publication March 27, 2007.) 